\title{
Ground-state phase diagram of the triangular lattice Hubbard model by the density-matrix renormalization group method
}

\author{
Tomonori Shirakawa ${ }^{1}$, Takami Tohyama ${ }^{2}$, Jure Kokalj ${ }^{3,4}$, Sigetoshi Sota ${ }^{5}$, and Seiji Yunoki ${ }^{1,5,6}$ \\ ${ }^{1}$ Computational Quantum Matter Research Team, \\ RIKEN Center for Emergent Matter Science (CEMS), Wako, Saitama 351-0198, Japan \\ ${ }^{2}$ Department of Applied Physics, Tokyo University of Science, Tokyo 125-8585, Japan \\ ${ }^{3}$ Jožef Stefan Institute, Jamova c. 39, 1000 Ljubljana, Slovenia \\ ${ }^{4}$ Faculty of Civil and Geodetic Engineering, University of Ljubljana, SI-1000 Ljubljana, Slovenia \\ ${ }^{5}$ Computational Materials Science Research Team, \\ RIKEN Advanced Institute for Computational Science (AICS), Kobe, Hyogo 650-0047, Japan \\ ${ }^{6}$ Computational Condensed Matter Physics Laboratory, RIKEN, Wako, Saitama 351-0198, Japan
}

(Dated: November 20, 2017)

\begin{abstract}
Two-dimensional density-matrix renormalization group method is employed to examine the ground state phase diagram of the Hubbard model on the triangular lattice at half-filling. The calculation reveals two discontinuities in the double occupancy with increasing the repulsive Hubbard interaction $U$ at $U_{\mathrm{c} 1} \sim 7.8 t$ and $U_{\mathrm{c} 2} \sim 9.9 t$ ( $t$ being the hopping integral), indicating that there are three phases separated by first order transitions. The absence of any singularity in physical quantities for $0 \leq U<U_{\mathrm{c} 1}$ implies a metallic phase in this regime. For $U>U_{\mathrm{c} 2}$, the local spin density induced by an applied pinning magnetic field exhibits a three sublattice feature, which is compatible with the $120^{\circ}$ Néel ordered state realized in the limit of $U \rightarrow \infty$. For $U_{\mathrm{c} 1}<U<U_{\mathrm{c} 2}$, a response to the applied pinning magnetic field is comparable to that in the metallic phase with a relatively large spin correlation length, but showing neither valence bond nor chiral magnetic order, which therefore resembles gapless spin liquid. However, the spin structure factor for the intermediate phase exhibits the maximum at the $K$ and $K^{\prime}$ points in the momentum space, which is not compatible to spin liquid with a large spinon Fermi surface. The calculation also finds that the pairing correlation function monotonically decreases with increasing $U$ and thus the superconductivity is unlikely in the intermediate phase.
\end{abstract}

\section{INTRODUCTION}

There have been accumulating experimental evidences that several organic materials, $\kappa$-(BEDT$\mathrm{TTF})_{2} \mathrm{Cu}_{2}(\mathrm{CN})_{3}$ [1], $\mathrm{EtMe}_{3} \mathrm{Sb}\left[\mathrm{Pd}(\mathrm{dmit})_{2}\right]_{2}$ [2-4], and $\kappa-\mathrm{H}_{3}$ (Cat-EDT-TTF $)_{2} \quad[5]$, forming a quasi twodimensional (2D) triangular structure and exhibit quantum spin liquid (QSL) [6, 7], where any spatial symmetry breaking does not occur due to the quantum fluctuation, even when it is cooled down to zero temperature. The realization of QSL against a symmetry-broken ordered state in higher spatial dimensions more than one dimension is one of the long standing issues in condensed matter physics [6] since the first proposal of resonating valence bonds (RVB) states by Anderson [8]. It has been considered that one of the key ingredients for the emergence of stable QSL is geometrical frustration [8], which increases quantum fluctuations and thus prevents symmetry breaking. In this context, the spin- $1 / 2$ antiferromagnetic Heisenberg model on the triangular lattice had been considered [9]. However, recent numerical studies, including two-dimensional density-matrix renormalization group (2D-DMRG) analysis, have suggested that the ground state of the spatially isotropic model is $120^{\circ}$ Néel ordered [10-13].

In addition to the geometrical frustration, other factors for stabilizing QSL have also been considered, such as i) the spatially anisotropic exchange interactions [14], ii) the higher order corrections of exchange interactions [15], and iii) the charge degree of freedom [16-19]. The later two are captured by the triangular lattice Hubbard model at half electron filling described by the Hamiltonian

$$
\mathcal{H}=-t \sum_{\langle i, j\rangle} \sum_{\sigma=\uparrow, \downarrow}\left(c_{i, \sigma}^{\dagger} c_{j, \sigma}+\text { h.c. }\right)+U \sum_{i} n_{i, \uparrow} n_{i, \downarrow},
$$

where $c_{i, \sigma}\left(c_{i, \sigma}^{\dagger}\right)$ represents the annihilation (creation) operator of an electron with spin $\sigma(=\uparrow, \downarrow)$ at site $i$ on the triangular lattice, $n_{i, \sigma}=c_{i, \sigma}^{\dagger} c_{i, \sigma}$, and the sum $\langle i, j\rangle$ runs over all pairs of nearest-neighbor sites $i$ and $j$. Indeed, the QSL phase in the organic materials appears next to the metallic phase, indicating that the QSL occurs close to the Mott metal-insulator transition [20-22] where the above two factors ii) and iii) are important. In fact, it has been extensively argued that the triangular lattice Hubbard model is the simplest effective model to describe and understand the metal-insulator transition and the QSL phase in the organic materials [23].

Elucidating the ground state phase diagram of the triangular lattice Hubbard model at half-filling is a challenge for numerical techniques in strongly correlated electron systems. Various numerical methods [24-43] have been applied so far, but the results are, nevertheless, controversial. The exact diagonalization techniques [24-26], the variational cluster approximation (VCA) [27, 28], the path-integral renormalization group (PIRG) method [29, 30], as well as the recently proposed ladder dual-Fermion approach [31] have suggested that there exist three phases in the ground state phase dia- 
gram with increasing $U / t$, i.e., a metallic phase, a nonmagnetic insulating phase, and the $120^{\circ}$ Néel ordered insulating phase. This is also supported by the numerical analysis of an effective strong coupling spin model [32]. On the other hand, the variational Monte Carlo methods [33-36] have suggested the absence of the nonmagnetic insulating phase. Besides the presence or absence of the intermediate insulating phase, the critical $U_{c} / t$ for the metal-insulator transition significantly varies among different methods [26].

Recently, the 2D-DMRG method has been applied to various 2D strongly correlated quantum systems [4461], although the DMRG method is best performed for one-dimensional gapful systems [62-65]. This is because the 2D-DMRG calculations with keeping large enough number of adapted density-matrix eigenstates to guarantee the desired numerical accuracy have become possible within reasonable computational resources, specially, for 2D spin- $1 / 2$ Heisenberg models [47].

Here, we employ the 2D-DMRG method to examine the ground state phase diagram of the repulsive Hubbard model on the triangular lattice at half electron filling. Our calculation reveals two discontinuities in the double occupancy of electrons with increasing $U / t$ at $U_{\mathrm{c} 1}=7.55 t \sim 8.05 t$ and $U_{\mathrm{c} 2}=9.65 t \sim 10.15 t$ for three different clusters up to 48 sites, strongly indicating that there are three phases separated by first order transitions at $U_{\mathrm{c} 1}$ and $U_{\mathrm{c} 2}$. The spin oscillation pattern for $U>U_{\mathrm{c} 2}$ under a pinning magnetic field exhibits a three sublattice feature, compatible with the $120^{\circ}$ Néel ordered state. Moreover, the spatial distribution of the nearest-neighbor spin correlation is found to be quite different among the three phases. The suppression of oscillatory behavior in the intermediate phase at $U_{\mathrm{c} 1}<U<U_{\mathrm{c} 2}$ suggests this phase in neither bond order nor valence bond solid. In addition, the spin correlation length in the intermediate phase is found to be larger than that for $U<U_{\mathrm{c} 1}$ but smaller than that for $U>U_{\mathrm{c} 2}$. Furthermore, the response to a pinning magnetic field in the intermediate phase is rather comparable to that in the paramagnetic metallic state. These features in the intermediate phase resembles gapless spin liquid [23, 66]. However, the spin structure factor in the intermediate phase shows a single maximum at the $\mathrm{K}$ and $\mathrm{K}^{\prime}$ points in the momentum space, which is not compatible with the expectation for the spinon Fermi sea state [15]. Superconductivity is also excluded in the intermediate phase.

The rest of this paper is organized as follows. First, the shape of $2 \mathrm{D}$ clusters studied here is introduced and the convergence of the DMRG calculations is discussed in Sec. II. Section III is devoted to our results for the triangular lattice Hubbard model at half-filling. We first show the ground state energy and the double occupancy to reveal the existence of three phases in Sec. III A. Next, we explore the properties of the ground state in each phase by calculating different quantities, including the response to a pinning magnetic field in Sec. III B, the spin correlation function in Sec. III C, the spin structure factor in
Sec. III D, the spatial distribution of the nearest-neighbor spin and bond correlations in Sec. III E and Sec. III F, respectively, the chiral correlation function in Sec. III G, and the pairing correlation function in Sec. IIIH. We then discuss possible relevance to the experimental observation and provide several remarks in Sec. IV, before summarizing the paper in Sec. V. In Appendix A, we examine the entanglement gap of the ground state as a function of $U / t$.

\section{METHOD}

We consider 32-, 36- and 48-site clusters depicted in Fig. 1. Since the results for these different clusters are qualitatively the same, we shall mainly show the results for the 36-site cluster. Following the notation in Refs. 58 and 59, clusters forming the triangular lattice can be classified as $\mathrm{XCn}(\mathrm{YCn} n)$ where the bond direction of a cluster is parallel to the $x$ direction ( $y$ direction), as shown in Fig. 1, and $n$ in $\mathrm{XCn}(\mathrm{YCn})$ represents the number of bonds in zigzag (vertical) $y$ direction. Accordingly, the 36- and 48-site clusters belong to XC6, and the 32-site cluster belongs to $\mathrm{YC} 4$. (a) 36-site cluster

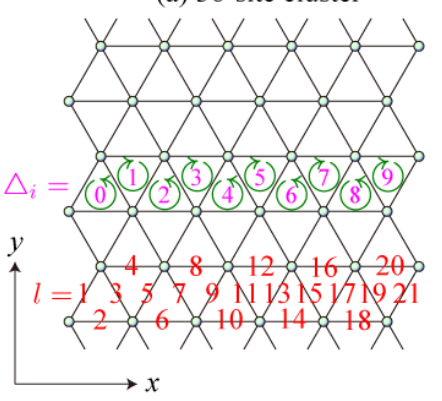

(b) 32-site cluster

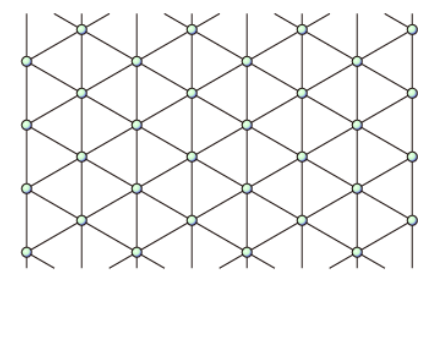

(c) 48-site cluster

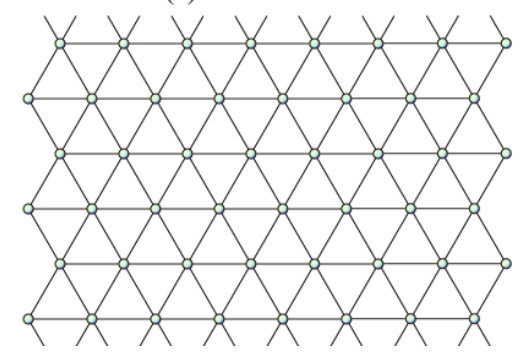

Figure 1. The (a) 36-, (b) 32-, and (c) 48-site clusters. Open (periodic) boundary conditions are imposed in the $x$ direction ( $y$ direction). The indexing of bonds $(l=1,2,3, \cdots, 21)$ as well as elementary triangles $\left(\triangle_{i}=0,1,2, \ldots, 9\right)$ with their chiral directions (arrows) are indicated in (a).

Figure 2 shows the convergence of the ground state energy for the 36-site cluster, as a function of the discarded weight $\delta_{m}$ defined as

$$
\delta_{m}=1-\sum_{n=1}^{m} \lambda_{n},
$$


where $\lambda_{n}$ is the $n$th largest eigenvalue of the reduced density-matrix of the ground state. As shown in Fig. 2, we find that the ground state energies for $m \geq 10000$ scale linearly with $\delta_{m}$, implying that the convergence of our calculations is well controlled.
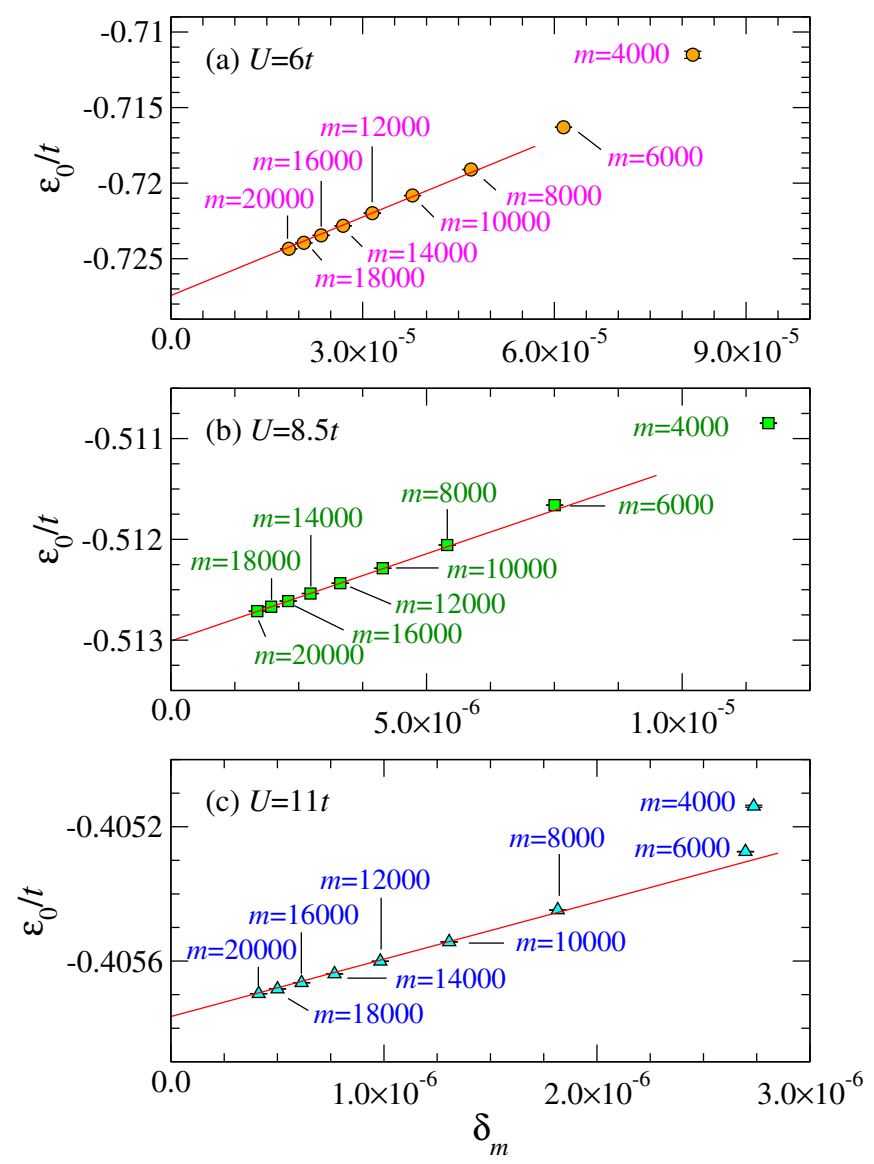

Figure 2. (color online) Ground state energy per site $\varepsilon_{0}$ as a function of the discarded weight $\delta_{m}$ for (a) $U=6 t$, (b) $U=8.5 t$, and (c) $U=11 t$. The cluster used here is the 36site cluster shown in Fig. 1(a). The number $m$ of eigenstates of the reduced density-matrix kept in the DMRG calculations is indicated beside each data point. A red straight line shows a linear fit to the three data points with $m=10$ 000, 12000 , and 14000 .

Throughout the study, we set the $z$ component of total spin to be zero. We keep up to $m=10000$ density-matrix eigenstates for the 32-site cluster, $m=14000$ for the 36site cluster, and $m=20000$ for the 48 -site cluster. As shown in Fig. 2, when we use $m=14000$ density-matrix eigenstates for the 36-site cluster, the typical orders of the discarded weight are $2.7 \times 10^{-5}$ for $U=6 t, 2.7 \times 10^{-6}$ for $U=8.5 t$, and $7.6 \times 10^{-7}$ for $U=11 t$. On the other hand, when we use $m=20000$ density-matrix eigenstates for the 48-site cluster, the typical orders of the discarded weight are $2.6 \times 10^{-5}$ for $U=6 t, 2.3 \times 10^{-6}$ for $U=$ $8.5 t$, and $2.8 \times 10^{-7}$ for $U=11$, thus obtaining the convergence similar to that for the 36 -site cluster.

\section{RESULTS}

\section{A. Energy and double occupancy}

We first study the $U / t$ dependence of the ground-state energy and double occupancy. Figures 3(a) and 3(b) show the ground-state energy per site,

$$
\varepsilon_{0}=\left\langle\psi_{0}|\mathcal{H}| \psi_{0}\right\rangle / N
$$

and the site average of the double occupancy,

$$
n_{d}=\frac{1}{N} \sum_{i}\left\langle\psi_{0}\left|n_{i, \uparrow} n_{i, \downarrow}\right| \psi_{0}\right\rangle
$$

where $\left|\psi_{0}\right\rangle$ is the ground-state obtained by the 2DDMRG calculation and $N$ is the number of sites. As shown in Fig. 3(b), there exist two discontinuities in the double occupancy. It should be noted that $\varepsilon_{0}$ and $n_{d}$ are related via $n_{d}=\partial \varepsilon_{0} / \partial U$. We have numerically verified this relation, supporting the satisfactory convergence of our results.

As shown in Fig. 3(c), the discontinuities in the double occupancy are most apparent when $n_{d} U^{2}$ is plotted. The first discontinuity occurs at $U_{\mathrm{c} 1} / t=7.55-8.05$ and the second one is located at $U_{\mathrm{c} 2} / t=9.65-10.15$. We find that these discontinuities in $n_{d} U^{2}$ become sharper with increasing $m$, indicating the nature of the first-order transition. We also find in the insets of Figs. 3(b) and 3(c) that there is no additional discontinuity for $0 \leq U<U_{\mathrm{c} 1}$. Therefore, we conclude that there exist two first order transitions separating three phases. In the following, we call the three regions phases I, II, and III, as indicated in Fig. 3.

Let us now compare our results with the previous studies. Since it includes the noninteracting limit with $U=0$, phase I is regarded as the metallic phase. The exact diagonalization analysis of a 16-site cluster using a finitetemperature Lanczos method has found that the metalinsulator transition occurs at $U_{\mathrm{c}} / t=7.5 \pm 0.5$ [26]. The metal-insulator transition is also found at $U_{\mathrm{c}} / t \sim 7.4 \pm 0.1$ for clusters up to 36 sites by the PIRG method [30]. These $U_{\mathrm{c}}$ values are rather similar to $U_{\mathrm{c} 1}$ in our calculations. On the other hand, the metal-insulator transition found by the VCA is at $U_{\mathrm{c}} / t \sim 6.3-6.7[27,28]$, which is slightly smaller than $U_{\mathrm{c} 1}$. This is probably due to smaller clusters used in these VCA calculations, which tend to enhance an insulating phase. We calculate in Appendix A the entanglement spectrum of the ground state as a function of $U / t$ and find an abrupt increase of the entanglement gap in the charge sector, supporting that the transition between phases I and II can be regarded as the metal-insulator transition.

The analysis based on the strong coupling expansion of the triangular lattice Hubbard model for clusters up to 36 sites [32] finds that the phase transition from the $120^{\circ}$ Néel-ordered phase to an insulating QSL phase occurs at $U_{\mathrm{c}} \sim 10 t$, which is close to $U_{\mathrm{c} 2}$ obtained in our calculations. The intermediate insulating phase with 


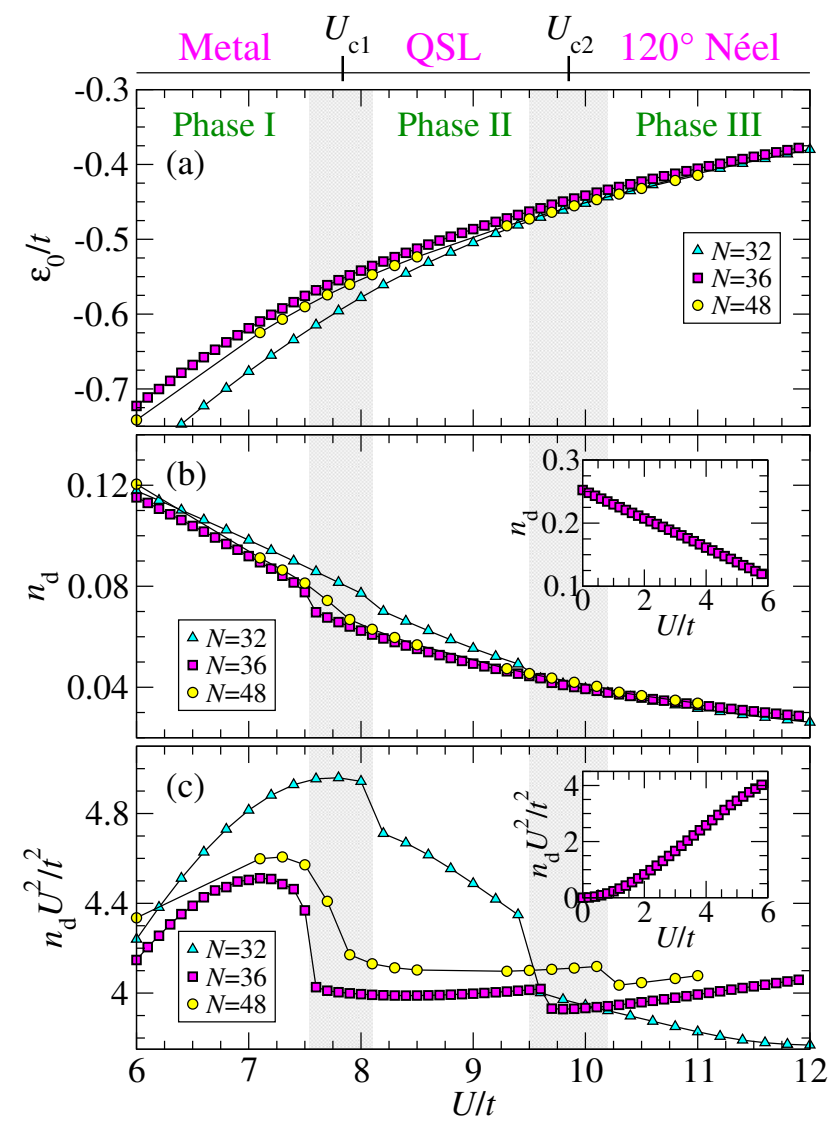

Figure 3. (color online) (a) Ground state energy per site $\epsilon_{0}$, (b) double occupancy $n_{d}$, and (c) $n_{d} U^{2}$ for three different clusters. Insets in (b) and (c) show the results for small values of $U$ calculated in the 36 -site cluster. The ground state phase diagram is shown schematically in the top panel, where QSL denotes quantum spin liquid. The phase boundaries are indicated by gray shades.

$U_{\mathrm{c} 2}=9.2 t \pm 0.3 t$ is also reported in the PIRG calculations [30]. Based on the comparison with these previous studies, phases II and III found in our calculations should correspond to a QSL phase and the $120^{\circ}$ Néel-ordered phase, respectively. In the following, we shall examine the nature of these phases.

\section{B. Response to a pinning magnetic field}

Let us first explore a possible magnetic order by applying a pinning magnetic field along the $z$-direction at a single site located at the edge of the cluster (see Fig. 4). The pinning magnetic field applied at site $i_{\text {imp }}$ is described by the following Hamiltonian:

$$
\mathcal{H}^{\prime}=-\sum_{i} h_{i} \mathcal{S}_{i}^{z},
$$

where $h_{i}=h \delta_{i, i_{\mathrm{imp}}}, \delta_{i, j}$ is the Kronecker delta (i.e., $\delta_{i, j}=$ 1 only when $i=j$ ), and $\mathcal{S}_{i}^{z}=\frac{1}{2}\left(n_{i, \uparrow}-n_{i, \downarrow}\right)$. The results of the local spin density

$$
S_{i}^{z}=\left\langle\psi_{0}\left|\mathcal{S}_{i}^{z}\right| \psi_{0}\right\rangle
$$

are summarized in Fig. 4. Note that the local spin density $S_{i}^{z}$ is zero in the absence of the pinning magnetic field. (a) $U=6 t$

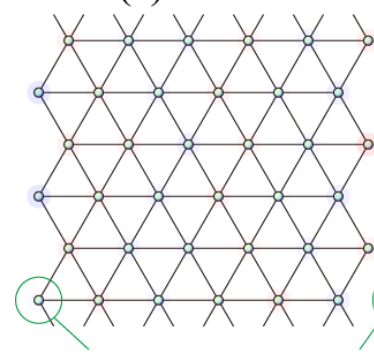

pinning magnetic field (b) $U=8.5 t$

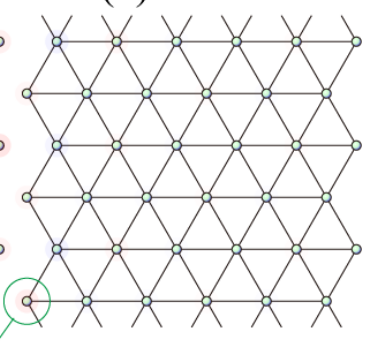

(d) Heisenberg model

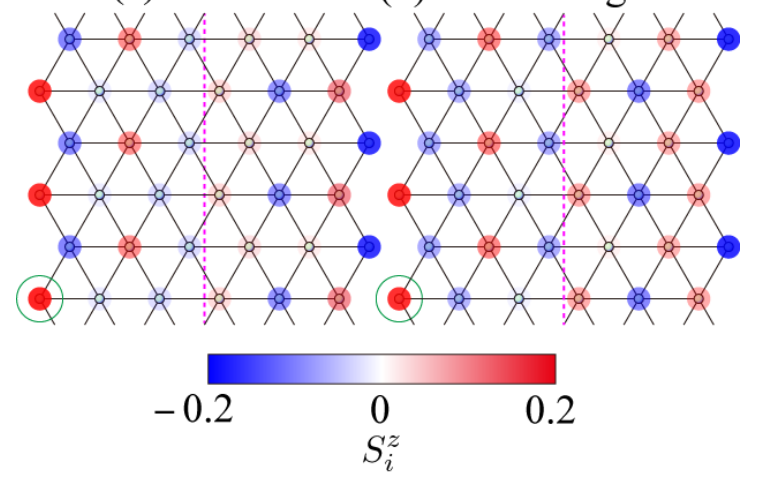

Figure 4. (color online) Local spin density $S_{i}^{z}$ for (a) $U=6 t$, (b) $U=8.5 t$, and (c) $U=11 t$ when a pinning magnetic field $h=0.005 t$ is applied along the $z$ direction at a single site located at the edge of the 36-site cluster (indicated by a green open circle). For comparison, the results for the spin- $1 / 2$ antiferromagnetic Heisenberg model on the triangular lattice with the nearest-neighbor exchange interaction $J$ is also shown in (d). Here, the same 36-site cluster is used as in (a)-(c) and the applied pinning magnetic field is $h=0.005 \mathrm{~J}$. The dashed line in (c) and (d) indicates a domain wall separating the cluster into two pieces, each of which exhibits a three sublattice pattern, expected for the $120^{\circ}$ Néel order. Note that the $z$ component of total spin is kept zero, i.e., $\sum_{i=1}^{N} S_{i}^{z}=0$, in the calculations.

As shown in Fig. 4(c), the local spin density for $U=$ $11 t$ in phase III exhibits a three sublattice pattern, compatible with the $120^{\circ}$ Néel order, except that there exists a domain wall at the center of the cluster running along the $y$ direction. Indeed, the spatial distribution of the local spin density found here, including the domain wall structure, is essentially identical to that for the spin-1/2 antiferromagnetic Heisenberg model on the triangular lattice with the nearest-neighbor exchange interaction [see Fig. 4 (d)], where the ground state is $120^{\circ}$ Néel ordered [10-13]. Therefore, this is strong evidence that the ground state in phase III is also $120^{\circ}$ Néel or- 
dered. In contrast, as shown in Figs. 4(a) and 4(b), the local spin densities for $U=6 t$ in phase I and $U=8.5 t$ in phase II are less affected by the pinning magnetic field, indicating the absence of long-range magnetic order.

Applying the perturbation theory, the leading correction of the spin density $\Delta S_{i}^{z}$ at site $i$ is

$$
\Delta S_{i}^{z} \sim-h \sum_{n(\neq 0)} \frac{\left\langle\psi_{0}\left|\mathcal{S}_{i_{\mathrm{imp}}}^{z}\right| \psi_{n}\right\rangle\left\langle\psi_{n}\left|\mathcal{S}_{i}^{z}\right| \psi_{0}\right\rangle}{E_{0}-E_{n}}
$$

where $\left|\psi_{n}\right\rangle$ is the $n$th eigenstate of $\mathcal{H}$ (without the pinning magnetic field) with its eigenvalue $E_{n}$. Since $\mathcal{H}$ commutes with the total spin operator, the total spin $S_{\text {tot }}$ is a good quantum number. We now assume that the ground state $\left|\psi_{0}\right\rangle$ is spin singlet with $S_{\text {tot }}=0$. Then, the Wigner-Eckert theorem states that the matrix elements in the numerator of Eq. (7) satisfy

$$
\left\langle\psi_{n}\left|\mathcal{S}_{i}^{z}\right| \psi_{0}\right\rangle=\left\{\begin{array}{cc}
0 & \text { if }\left|\psi_{n}\right\rangle \notin S_{\mathrm{tot}}=1 \\
\text { finite } & \text { if }\left|\psi_{n}\right\rangle \in S_{\mathrm{tot}}=1
\end{array}\right.
$$

where $\left|\psi_{n}\right\rangle \in S_{\text {tot }}=1\left(\left|\psi_{n}\right\rangle \notin S_{\text {tot }}=1\right)$ indicates that $\left|\psi_{n}\right\rangle$ belongs (does not belong) to the $S_{\text {tot }}=1$ subspace. Therefore, the different behavior of the local spin density under the applied pinning magnetic field should be attributed to the amount of the low-lying triplet excitations.

(a) $U=6 t$

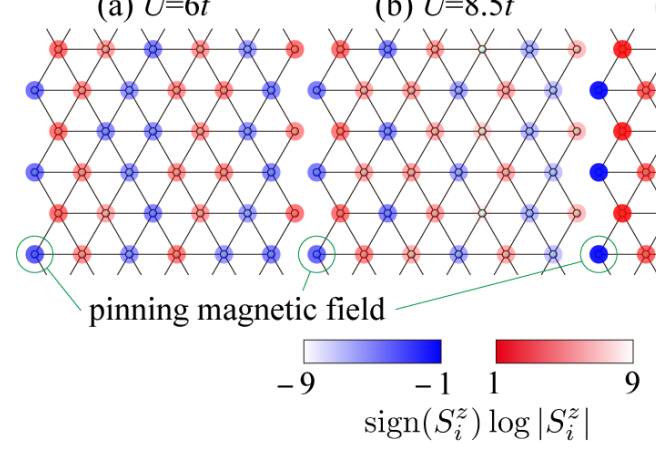

Figure 5. (color online) Same as Figs. 4(a)-4(c) but in the logarithmic scale.

Figure 5 shows the same result as in Fig. 4 but in the logarithmic scale. It clearly shows that the local spin density $S_{i}^{z}$ for $U=8.5 t$ in phase II exhibits comparable amplitude with that for $U=6 t$ in phase I where the ground state is the paramagnetic metal and thus no triplet excitation gap is expected. On the other hand, the local spin density $S_{i}^{z}$ for $U=8.5 t$ is significantly smaller than that for $U=11 t$ in phase III where the ground state is $120^{\circ}$ Néel ordered. The similarity to the $U=6 t$ case thus indicates that there exist an extensive amount of gapless spin excitations in phase II expected in the thermodynamic limit.

\section{Spin correlation function}

Next, we calculate the spin correlation

$$
S_{i, j}=\left\langle\psi_{0}\left|\mathcal{S}_{i}^{z} \mathcal{S}_{j}^{z}\right| \psi_{0}\right\rangle
$$

between a reference site $j$ located at the center of the cluster and other sites $i$. The representative results for the three different phases are shown in Fig. 6. Figure 6(c) clearly shows that $S_{i, j}$ for $U=11 t$ in phase III exhibits a three sublattice pattern, compatible with the $120^{\circ}$ Néel order. On the other hand, $S_{i, j}$ in phases I and II does not show such a three sublattice pattern [see Figs. 6(a) and $6(\mathrm{~b})$ ], strongly suggesting that these phases are not $120^{\circ}$ Néel ordered. (a) $U=6 t$

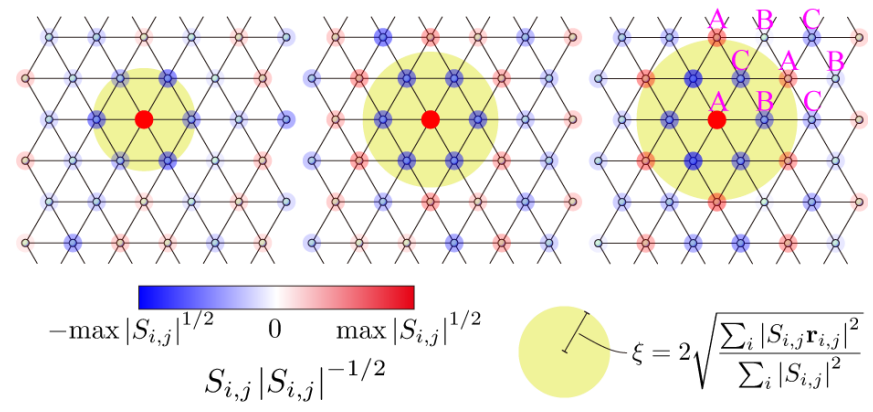

Figure 6. (color online) Spin correlation function $S_{i, j} / \sqrt{\left|S_{i, j}\right|}$ between a reference site $j$ at the center of the cluster (indicated by a red circle) and other sites $i$ for (a) $U=6 t$, (b) $U=8.5 t$, and (c) $U=11 t$. Here, $\max \left|S_{i, j}\right|^{1 / 2}$ represents the maximum value of $\left|S_{i, j}\right|^{1 / 2}$ for each $U$. Yellow-green shaded circle indicates the correlation length $\xi$. A three sublattice pattern expected for the $120^{\circ}$ Néel order is indicated in (c) by "A," "B," and "C," where $S_{i, j}>0\left(S_{i, j}<0\right)$ if sites $i$ and $j$ belong to the same (different) sublattice. The cluster used here is the 36-site cluster shown in Fig. 1(a).

We notice, however, in Fig. 6 that the intensity of $S_{i, j}$ between distant sites for $U=8.5 t$ is comparable to that for $U=11 t$, indicating the relatively long spin correlation in phase II. For a quantitative comparison, we estimate the correlation length $\xi$ via

$$
\xi=2 \sqrt{\frac{\sum_{i=1}^{N}\left|S_{i, j} \mathbf{r}_{i, j}\right|^{2}}{\sum_{i=1}^{N}\left|S_{i, j}\right|^{2}}},
$$

where $\mathbf{r}_{i, j}=\mathbf{r}_{i}-\mathbf{r}_{j}$ and $\mathbf{r}_{i}$ is the position vector of site $i$. As shown in Fig. 6, we find that $\xi$ for $U=8.5 t$ is shorter than that for $U=11 t$ where $\xi$ diverges in the thermodynamic limit, but is longer than that for $U=$ $6 t$ where the spin correlation decays algebraically. The rather long range spin correlation in phase II should be contrasted with the exponential decay of spin correlation expected in gapped QSL. Recalling also the result of the response to the pinning magnetic field, the ground state in phase II resembles gapless QSL. 


\section{Spin structure factor}

The spin structure factor $S(\mathbf{q})$ is the Fourier transform of the real-space spin correlation function $S_{i, j}$ and defined as

$$
S(\mathbf{q})=\sum_{i=1}^{N} S_{i, j} e^{\mathrm{i} \mathbf{q} \cdot\left(\mathbf{r}_{i}-\mathbf{r}_{j}\right)},
$$

where site $j$ is a representative site chosen at the central site of the cluster as in Fig. 6. Although the wave number $\mathbf{q}$ is not a good quantum number due to open boundary conditions in the $x$ direction, here we calculate $S(\mathbf{q})$ for arbitrary $\mathbf{q}$.

The representative results for the three different phases are shown in Figs. 7(a)-7(c). We find in Fig. 7(c) that $S(\mathbf{q})$ for $U=11 t$ in phase III displays sharp peaks at $\mathbf{q}=(2 \pi / 3,2 \pi / \sqrt{3})$ (the $\mathrm{K}$ point) and other equivalent q's including the $\mathrm{K}^{\prime}$ point at $\boldsymbol{q}=(-2 \pi / 3,2 \pi \sqrt{3})$, which is compatible with the $120^{\circ}$ Néel-ordered state. The $S(\mathbf{q})$ for $U=8.5 t$ in phase II shown in Fig. 7(b) also exhibits broad maxima at the $\mathrm{K}$ point and other equivalent q's, but the peak structure is softened as compared with $S(\mathbf{q})$ for $U=11 t$. In contrast, we find in Fig. $7($ a) that $S(\mathbf{q})$ for $U=6 t$ in phase I shows enhanced intensities forming a ring-like structure around the $\mathrm{K}$ point (and other equivalent q's), which implies the presence of $2 \mathbf{k}_{\mathrm{F}}$ scattering, where $\mathbf{k}_{\mathrm{F}}$ is the Fermi momentum in the noninteracting limit.

In order to better understand $S(\mathbf{q})$ in phases I and II, we calculate $S(\mathbf{q})$ within the random phase approximation (RPA). In the RPA, the spin susceptibility $\chi(\mathbf{q}, z)$ at zero temperature is given as

$$
\chi(\mathbf{q}, z)=\frac{\chi_{0}(\mathbf{q}, z)}{1-U \chi_{0}(\mathbf{q}, z)},
$$

where $z$ is the complex frequency. The susceptibility $\chi_{0}(\mathbf{q}, z)$ in the noninteracting limit is

$$
\chi_{0}(\mathbf{q}, z)=\frac{1}{N} \sum_{\mathbf{k} \in 1 \mathrm{st} \mathrm{BZ}} \frac{\Theta\left(-\varepsilon_{\mathbf{k}}\right)-\Theta\left(-\varepsilon_{\mathbf{k}+\mathbf{q}}\right)}{z-\varepsilon_{\mathbf{k}}+\varepsilon_{\mathbf{k}+\mathbf{q}}},
$$

where the sum is taken over the first Brillouin zone (BZ) of the triangular lattice, $\Theta(x)$ is the Heaviside step function, and $\varepsilon_{\mathbf{k}}$ is the noninteracting band dispersion

$$
\begin{aligned}
\varepsilon_{\mathbf{k}}= & -2 t \cos k_{x}-2 t \cos \left(\frac{k_{x}}{2}+\frac{\sqrt{3} k_{y}}{2}\right) \\
& -2 t \cos \left(-\frac{k_{x}}{2}+\frac{\sqrt{3} k_{y}}{2}\right)-\mu .
\end{aligned}
$$

The chemical potential $\mu$ is tuned such that the electron density is 0.5 per spin. We set the chemical potential $\mu \sim 0.8347 t$ for the calculation in the thermodynamic limit.

From $\chi(\mathbf{q}, z)$ obtained above within the RPA, the spin structure factor $S(\mathbf{q})$ is evaluated as

$$
S(\mathbf{q})=\frac{1}{\pi} \int_{0}^{\infty} \mathrm{d} x \operatorname{Re} \chi(\mathbf{q}, \mathrm{i} x) .
$$
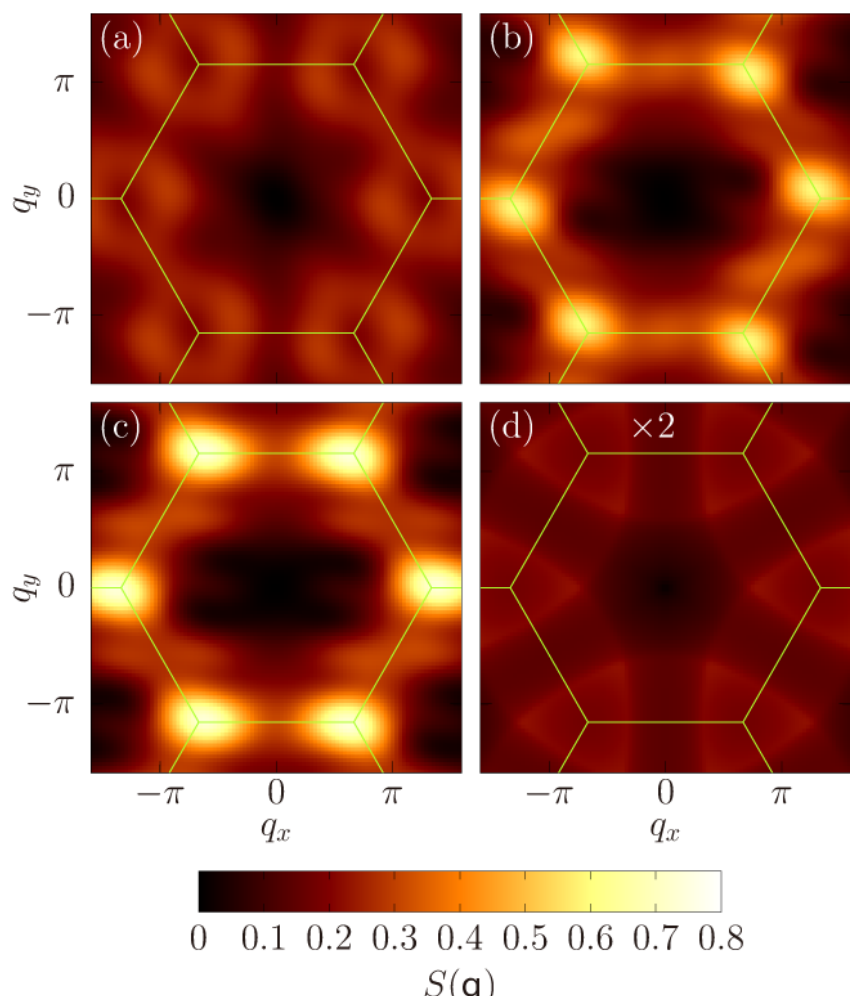

Figure 7. (color online) Intensity plot of spin structure factor $S$ (q) for (a) $U=6 t$, (b) $U=8.5 t$, and (c) $U=11 t$ calculated in the 36-site cluster and for (d) $U=3 t$ obtained by the random phase approximation. Notice that the intensity is doubled in (d) for clarity. The Brillouin zone boundaries are indicated by yellow-green lines.

In deriving the above equation, we have assumed that the $z$ component of total spin is zero and the system is invariant under the global spin flip. Figure $7(\mathrm{~d})$ shows $S(\mathbf{q})$ for $U=3 t$ within the RPA. Here we choose relatively small $U$ because $\chi(\mathbf{q}, 0)$ diverges at $U=3.7-3.8 t$. As shown in Fig. $7(\mathrm{~d}), S(\mathbf{q})$ exhibits a triangular shelllike structure around the $\mathrm{K}$ point (and other equivalent q's). The ridges of the shells lie exactly along the $2 \mathbf{k}_{\mathrm{F}}$ lines and the local minimum in the center of the shell is located at the K point (and other equivalent q's). These features are indeed similar to those found in Fig. 7(a) for $U=6 t$.

The spinon Fermi sea state is a kind of gappless spin liquid state and has been considered as a candidate for the ground state of triangular lattice systems [15]. Due to the presence of spinon Fermi surface, $S(\mathbf{q})$ for the spinon Fermi sea state exhibits singularities along the $2 \mathbf{k}_{\mathrm{F}}$ lines [67] and is expected to be similar to those shown in Figs. 7(a) and 7(d). However, as shown in Fig. 7(b), we find that $S(\mathbf{q})$ for $U=8.5 t$ in phase II is quite different from those in Figs. $7(\mathrm{a})$ and $7(\mathrm{~d})$. Therefore, the spinon Fermi sea state is unlikely to be the ground state in phase II.

The similarity of $S(\mathbf{q})$ for $U=8.5 t$ and $U=11 t$ in 
Fig. 7 tempts us to conclude that also the ground state in phase II shows tendency towards the $120^{\circ}$ Néel order. However, we emphasize that the peak structure in $S(\mathbf{q})$ for $U=8.5 t$ in phase II are much smaller and broader than that for $U=11 t$ in phase III. Indeed, as shown in Fig. 4 and Fig. 6, the response to the pinning magnetic field and the real-space spin correlation function are clearly different in phases II and III.

\section{E. Nearest-neighbor spin correlation}

We calculate the nearest-neighbor spin correlation $S_{\langle i, j\rangle}\left(=S_{i, j}\right)$ for all nearest-neighbor sites $i$ and $j$, and the representative results for the three different phases are shown in Fig. 8. We first notice in Figs. 8(a)-8(c) that the results are invariant under the translation along the $y$ direction, the reflection about mirror planes perpendicular to the $y$ direction, and the $180^{\circ}$ rotation around the center of the cluster, thus implying that the convergence of our results is satisfactory. For better quantitative comparison, we show in Fig. 8(d) $S(l)=S_{\langle i, j\rangle}$ along the $x$ direction, where the bond index $l$ connecting sites $i$ and $j$ is denoted in Fig. 1(a).

(a) $U=6 t$

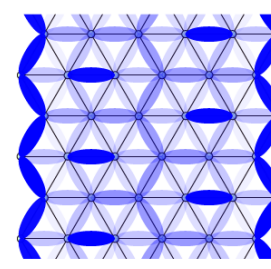

(b) $U=8.5 t$
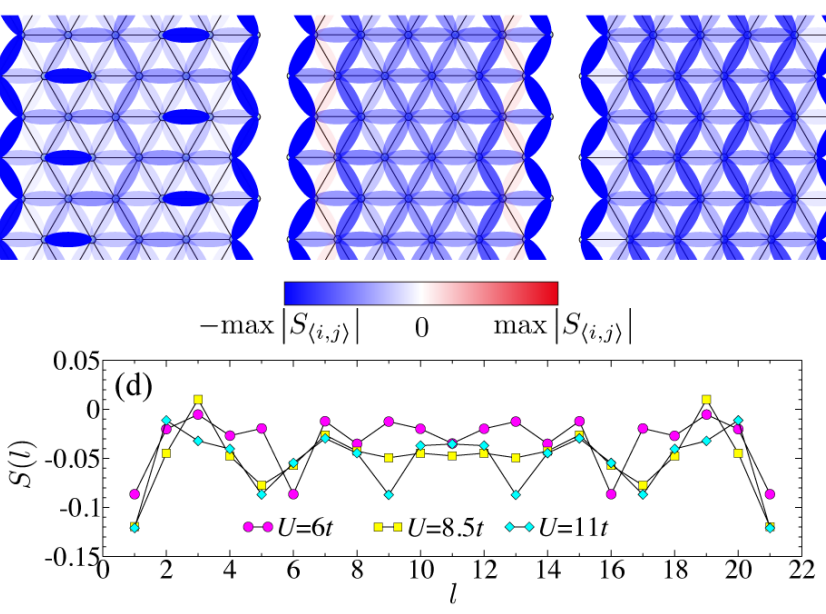

Figure 8. (color online) Nearest-neighbor spin correlation $S_{\langle i, j\rangle}$ for (a) $U=6 t$, (b) $U=8.5 t$, and (c) $U=11 t$. Here, $\max \left|S_{\langle i, j\rangle}\right|$ represents the maximum value of $\left|S_{\langle i, j\rangle}\right|$ for each $U$. (d) $S(l)=S_{\langle i, j\rangle}$ along $x$-direction, where the bond index $l$ connecting neighboring sites $i$ and $j$ is shown in Fig. 1(a). The 36-site cluster is used for all figures.

It is clearly observed in Fig. 8(d) that $S(l)$ for $U=11 t$ in phase III is rather enhanced at $l=4 n-3$ and suppressed at $l=4 n-1$ for $n=1,2,3, \cdots$. More interestingly, the oscillations in $S(l)$ for $U=8.5 t$ in phase II are smallest, especially around the center of the cluster. Note that spatial variation of $S(l)$ is an indication of spatial symmetry breaking, e.g., valence bond solid, or a tendency for it, and that in our case the spatial vari- ation is induced by open boundary conditions in the $x$ direction. The strong suppression of oscillations in phase II is therefore a strong indication of the absence of valence bond solid and also other possible spatial symmetry breakings.

Let us now compare the nature of the ground state in phase II with the $Z_{2}$ spin liquid ground state of the spin$1 / 2$ antiferromagnetic Heisenberg model on the triangular lattice with the next nearest-neighbor exchange interaction, recently reported in Refs. 58 and 59. The cluster used here (Fig. 1) is an odd cylinder [58, 59] with odd number of sites in the one dimensional unit cell. Therefore, according to the Lieb-Schultz-Mattis theorem [68], the ground state for this cluster is degenerate if the excitation gap is finite as in the case for the $Z_{2}$ spin liquid. Reflecting this degeneracy, the spatial distribution of the nearest-neighbor spin correlation $S_{\langle i, j\rangle}$ exhibits the strong alternating oscillation, induced by open boundary conditions along the $x$ direction $[58,59]$. Although the similar oscillation pattern is found in phase III and around the edge of the cluster in phase II, the central region of the cluster in phase II does not show such feature (see Fig. 8).

\section{F. Nearest-neighbor bond correlation}

We also calculate the hopping amplitude $B_{\langle i, j\rangle}$ between nearest-neighbor sites $i$ and $j$ defined as

$$
B_{\langle i, j\rangle}=\frac{1}{2}\left\langle\psi_{0}\left|\left(c_{i, \sigma}^{\dagger} c_{j, \sigma}+c_{j, \sigma}^{\dagger} c_{i, \sigma}\right)\right| \psi_{0}\right\rangle .
$$

The representative results for the three different phases are shown in Fig. 9. Similarly to $S_{\langle i, j\rangle}$, we find that $B_{\langle i, j\rangle}$ is invariant under the translation, reflection, and rotation operations [see Figs. 9(a)-9(c)], suggesting that our results are well converged. For better quantitative comparison, Fig. $9(\mathrm{~d})$ shows $B(l)=B_{\langle i, j\rangle}$ along the $x$ direction for the $l$ th bond connecting sites $i$ and $j$ [for the indexing of bonds, see Fig. 1(a)].

We find that $B_{\langle i, j\rangle}$ exhibits the similar oscillation patterns to those observed in $S_{\langle i, j\rangle}$ (see Fig. 8). The similarity between $B_{\langle i, j\rangle}$ and $S_{\langle i, j\rangle}$ is expected for large $U / t$ since the kinetic energy, proportional to $B_{\langle i, j\rangle}$, can be in a strong coupling regime transferred to the Heisenberg exchange interaction, which is related to $S_{\langle i, j\rangle}$. However, it is surprising that this similarity is present also in the coupling regimes shown in Fig. 9, where there is in general no direct connection between $B_{\langle i, j\rangle}$ and $S_{\langle i, j\rangle}$. As shown in Fig. 9(d), we find that the oscillations of $B(l)$ around the center of the cluster are most strongly reduced for $U=8.5 t$ in phase II as compared with those in phases I and III. This implies that the ground state in phase II is not compatible with the nearest-neighbor valence bond solid. 
(a) $U=6 t$
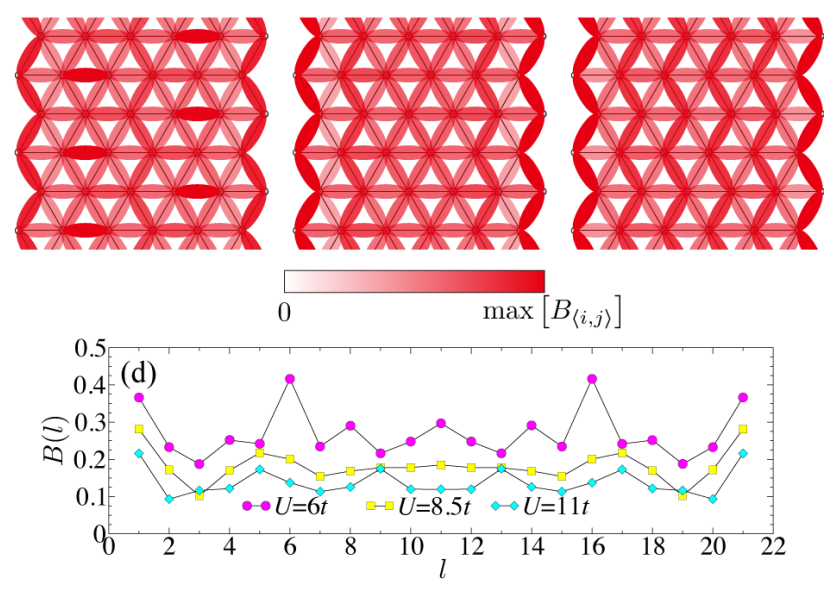

Figure 9. (color online) Nearest-neighbor hopping amplitude $B_{\langle i, j\rangle}$ for (a) $U=6 t$, (b) $U=8.5 t$, and (c) $U=11 t$. Here, $\max \left[B_{\langle i, j\rangle}\right]$ is the maximum value of $B_{\langle i, j\rangle}$ for each $U$. (d) $B(l)=B_{\langle i, j\rangle}$ along the $x$ direction, where the bond index $l$ connecting neighboring sites $i$ and $j$ is denoted in Fig. 1(a). Notice that the increase of kinetic energy, proportional to $-t \sum_{l} B(l)$, is nicely observed with increasing $U / t$. The 36site cluster is used for all figures.

\section{G. Chiral correlation function}

Next, we calculate the chiral correlation function $C\left(\triangle_{i}, \triangle_{j}\right)$ defined as

$$
C\left(\triangle_{i}, \triangle_{j}\right)=\left\langle\psi_{0}\left|C_{\triangle_{i}} C_{\triangle_{j}}\right| \psi_{0}\right\rangle
$$

with

$$
C_{\triangle_{i}}=\overrightarrow{\mathcal{S}}_{i_{1}} \cdot\left(\overrightarrow{\mathcal{S}}_{i_{2}} \times \overrightarrow{\mathcal{S}}_{i_{3}}\right)
$$

where $\triangle_{i}$ indicates the $i$ th elementary triangle formed by three neighboring sites $i_{1}, i_{2}$, and $i_{3}$ in clockwise or counter clockwise order, and the indexing of elementary triangles as well as their chiral directions is indicated in Fig. 1(a). $\vec{S}_{i}$ is the spin operator at site $i$ defined as

$$
\overrightarrow{\mathcal{S}_{i}}=\frac{1}{2} \sum_{\sigma_{1}=\uparrow, \downarrow} \sum_{\sigma_{2}=\uparrow, \downarrow} c_{i, \sigma_{1}}^{\dagger} \vec{\sigma}_{\sigma_{1}, \sigma_{2}} c_{i, \sigma_{2}},
$$

where $\vec{\sigma}=\left(\sigma_{x}, \sigma_{y}, \sigma_{z}\right)$ are Pauli matrices. Figure 10 shows the chiral correlation function $C\left(\triangle_{i}, \triangle_{i}+l\right)=C(l)$ with $\triangle_{i}=0$ along the $x$ direction [see Fig. 1(a)]. We find that the sign of $C(l)$ exhibits nontrivial oscillation [Fig 10(a)] and the amplitude of $C(l)$ decays exponentially [Fig 10(b)]. Therefore, we conclude that the chiral spin liquid is most unlikely to be the ground state in phase II [53].

\section{H. Pairing correlation function}

Finally, let us discuss the possibility of superconductivity by calculating the pairing correlation function

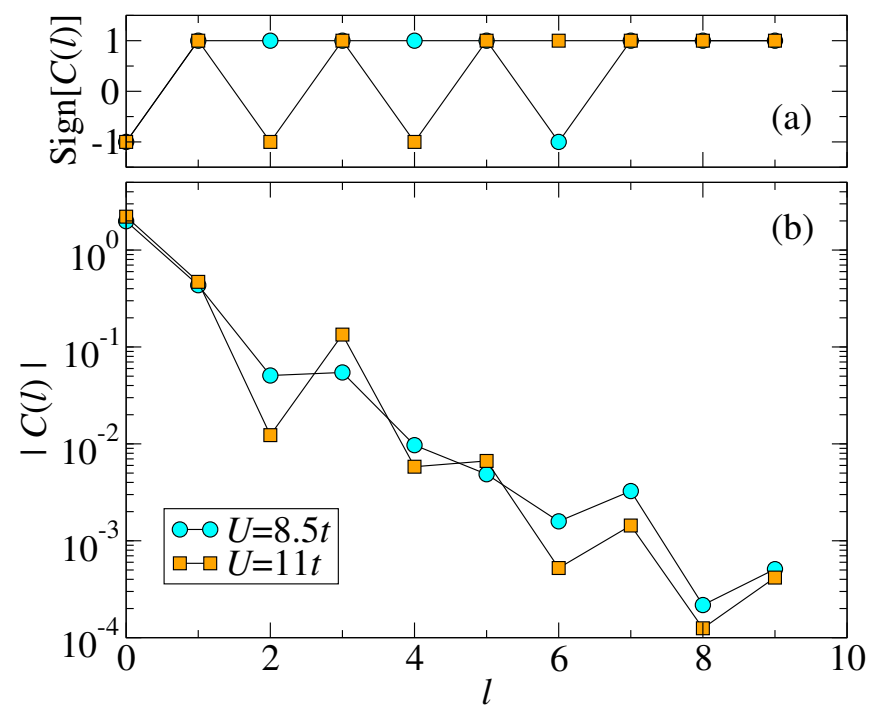

Figure 10. (color online) (a) Sign and (b) amplitude of chiral correlation function $C(l)$ for $U=8.5 t$ and $11 t$. The cluster used here is the 36-site cluster shown in Fig. 1(a).

$P_{\nu}(i, j, k, l)$ defined as

$$
P_{\nu}(i, j, k, l)=\left\langle\psi_{0}\left|\Delta_{\nu}(i, j) \Delta_{\nu}^{\dagger}(k, l)\right| \psi_{0}\right\rangle
$$

with the nearest-neighbor singlet channel $(\nu=\mathrm{s})$

$$
\Delta_{\mathrm{s}}(i, j)=\frac{1}{\sqrt{2}}\left(c_{i, \uparrow} c_{j, \downarrow}-c_{i, \downarrow} c_{j, \uparrow}\right)
$$

and the nearest-neighbor triplet channel $(\nu=\mathrm{t})$

$$
\Delta_{\mathrm{t}}(i, j)=\frac{1}{\sqrt{2}}\left(c_{i, \uparrow} c_{j, \downarrow}+c_{i, \downarrow} c_{j, \uparrow}\right) .
$$

Figure 11 shows the representative results of the pairing correlation function $P_{\nu}(r)=P_{\nu}(i, j, k, l)$ for both singlet and triplet channels, where $r$ is the distance between the centers of two pairs of nearest-neighbor sites $(i, j)$ and $(k, l)$. We find that the pairing correlations in both channels are significantly suppressed for $U=8.5 t$ in phase II and $U=11 t$ in phase III as compared with those for $U=6 t$ in phase I. Therefore, we conclude that the ground-state in phase II is unlikely to be superconducting. It should also be noted that the short range superconducting correlations in the spin triplet channel is stronger than those in the spin singlet channel for the three representative cases, although the superconducting correlations in the spin singlet channel dominates in the long distance.

It is also interesting to notice that the pairing correlations at long distances seem to be saturated for $U=6 t$ in phase I. However, since the longest distances are calculated from sites close to the cluster edges, the upturn of the pairing correlations might be a finite size effect. Therefore, our calculations alone cannot support 


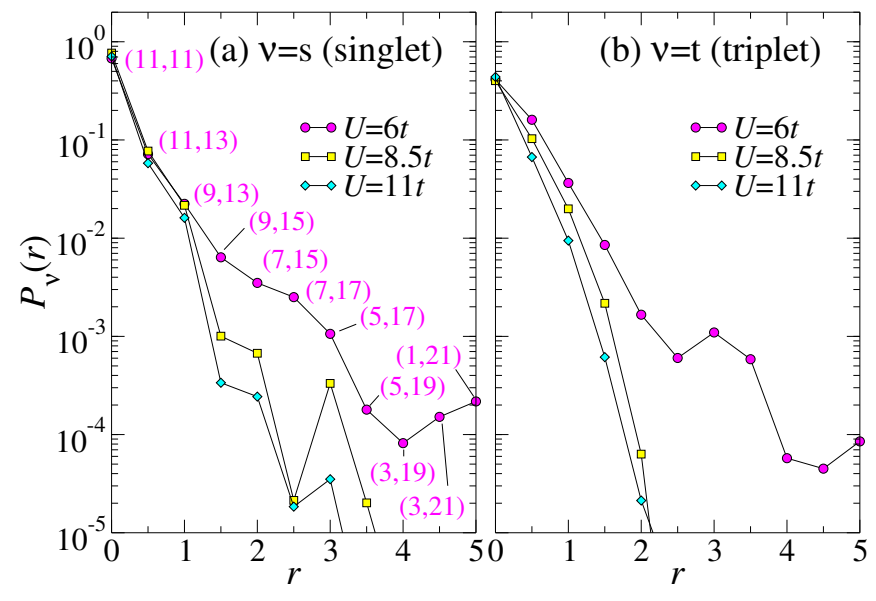

Figure 11. (color online) (a) Singlet $(\nu=\mathrm{s})$ and (b) triplet $(\nu=\mathrm{t})$ pairing correlation function $P_{\nu}(r)=P_{\nu}(i, j, k, l)$ for $U=6 t, 8.5 t$, and $11 t$ calculated in the 36 -site cluster. A pair of integer numbers $\left(l_{1}, l_{2}\right)$ beside each data point for $U=6 t$ denotes a pair of bond indexes $l_{1}$ and $l_{2}$ [for the definition, see Fig. 1(a)], representing nearest-neighbor sites $(i, j)$ and $(k, l)$, respectively, for which the pairing operators $\Delta_{\nu}(i, j)$ and $\Delta_{\nu}^{\dagger}(k, l)$ are chosen in Eq. (20). $r$ is the distance between the centers of bonds $l_{1}$ and $l_{2}$.

the presence of the superconducting phase. Larger systems with $U$ closer to $U_{\mathrm{c} 1}$ might show stronger pairing correlations. This issue is left for the future study.

\section{DISCUSSION}

Let us briefly discuss implications of our results for the experiments. Our results show a rather small discontinuity $\Delta n_{d}(\sim 0.007)$ in the double occupancy found at the metal-insulator transition $U_{\mathrm{c} 1}$, which is in sharp contrast to the previous studies using other approaches [30], where $\Delta n_{d}$ is typically much larger (from 0.02 to 0.06 ), and is even qualitatively different from the continuous transition discussed in Refs. 69 and 70. Smallness of the jump might be the origin of the controversy about the order of the transition. We note, however, that the discontinuity $\Delta n_{d}$ corresponds to the interaction (or equivalently kinetic) energy jump of $U_{\mathrm{c} 1} \Delta n_{d} \sim 0.05 t$, giving $\sim 25 \mathrm{~K}$ for the organic materials with $t \sim 50 \mathrm{meV}$ [26], which nicely compares with the temperature where the first-order transition line ends in the phase diagram of the organic materials [22].

Next, let us discuss the ground-state in phase II in terms of RVB states described by Gutzwiller projected fermionic wave functions. For this purpose, it is important to recall that the structure factor $S(\boldsymbol{q})$ in phase II exhibits the maximum at the $\mathrm{K}$ and $\mathrm{K}^{\prime}$ points. This feature is not consistent with a projected Fermi sea with a large Fermi surface because the $2 \mathbf{k}_{\mathrm{F}}$ structure is not found in $S(\boldsymbol{q})$. Instead, this feature is rather comparable to a projected Fermi sea with gapless nodal points such as a projected Dirac fermion [71, 72].

Another interesting feature is that the superconducting fluctuations for the spin-triplet (singlet) channel dominates in the short (long) distance, although the superconducting correlation functions for both channels decay exponentially in the insulating phases. This suggests that the long wavelength behavior of the ground-state in phase II might be captured by a projected BCS wave function with a singlet pairing, but the strong modification of the wave function would be required to describe the shortrange properties such as the ground-state energy.

\section{SUMMARY}

In summary, we have performed large scale 2D-DRMG calculations, using up to $m=20000$ density-matrix eigenstates, to examine the ground state phase diagram of the Hubbard model on the triangular lattice at halffilling. We have shown that the convergence of our results is well controlled and, therefore, our results can be regarded as the most accurate and unbiased results available at a moment, apart from the small cluster shape and size dependence.

We have found two first-order transitions separating the three different phases, which include the metallic phase in weak coupling region, the $120^{\circ}$ Néel-ordered phase in strong coupling region, and the QSL like phase in the intermediate couplin region. The weak and intermediate coupling phases are less affected by the pinning magnetic fields, suggesting the absence of magnetic order in these two phases. The spin correlations in the intermediate phase is weaker than those in the $120^{\circ}$ Néel-ordered phase and stronger than those in the metallic phase. The spin structure factor in the intermediate phase shows a maximum at the $\mathrm{K}$ and $\mathrm{K}^{\prime}$ points, which is not compatible with the spinon Fermi sea state [15]. The spatial distribution of the nearest-neighbor spin correlation in the intermediate phase is not comparable with the $Z_{2}$ spin liquid found in the spin-1/2 antiferromagnetic Heisenberg model on the triangular lattice with the next-nearestneighbor exchange interaction $[58,59]$. We have also calculated the chiral correlation function and found that the chiral spin liquid [53] is unlikely in the intermediate phase. The pairing correlation function decreases monotonically with increasing $U / t$, suggesting that the superconductivity is also unlikely in the intermediate phase.

The clusters used here are much smaller than those employed for the 2D-DMRG studies of spin- $1 / 2$ antiferromagnetic Heisenberg models on the triangular lattice reported in Refs. 58 and 59. This is simply because the local degrees of freedom in the Hubbard model is two times larger than those in the spin- $1 / 2$ Heisenberg models. Therefore, the more detail analysis using larger clusters is highly desirable in order to determine the nature of the ground state in the intermediate phase and, in particular, to address the size of the spin gap in the thermodynamic limit and the experimental observa- 
tion in $\mathrm{EtMe}_{3} \mathrm{Sb}\left[\mathrm{Pd}(\mathrm{dmit})_{2}\right]_{2}$ where gapless QSL is suggested [3]. Further properties of the intermediate phase, including the nature of excitations, remain to be firmly examined since that would greatly improve our understanding of spin liquid in general as well as of the organic materials in particular.

\section{ACKNOWLEDGMENTS}

The authors are grateful to S. Nishimoto and T. Li for valuable discussion. This work has been supported by Grant-in-Aid for Scientific Research from the Japan Society for the Promotion of Science (JSPS) (Grant No. 24740269, No. 26287079, and No. 17K14148) and Slovenian Research Agency (Z1-5442), and in part by RIKEN Molecular Systems and RIKEN iTHES Project. T. T. and J. K. acknowledge the visiting program for young researchers at Yukawa Institute for Theoretical Physics, Kyoto University. The computation has been performed using the RIKEN Cluster of Clusters (RICC), the RIKEN supercomputer system (HOKUSAI GreatWave), and the $\mathrm{K}$ computer at RIKEN Advanced Institute for Computational Science (AICS) under MEXT HPCI Strategic Programs for Innovative Research (SPIRE) (Project Nos. hp120137, hp140128, hp150112, hp160122, and hp170324).

\section{A: Entanglement Gap}

In this appendix, we examine the charge and spin gaps in the low-lying entanglement spectrum of the ground state [73] to support our results in the main text. In the DMRG method, the system is divided into two regions, blocks $A$ and $B$, and thus the ground state $|\psi\rangle$ is represented as

$$
|\psi\rangle=\sum_{i, j} \psi_{i j}|i\rangle_{A}|j\rangle_{B}
$$

where $|i\rangle_{A}\left(|j\rangle_{B}\right)$ denotes a basis in block $A(B)$. The reduced density matrix $\rho_{A}$ for block $A$ is obtained by tracing out the degrees of freedom in block $B$,

$$
\rho_{A}=\operatorname{Tr}_{B}|\psi\rangle\langle\psi|,
$$

where $\operatorname{Tr}_{B}$ indicates the trace over all bases in block $B$. The entanglement spectrum $\xi_{n}$ (where $n=1,2,3, \ldots$ ) is defined as

$$
\xi_{n}=-\ln \lambda_{n},
$$

where $\lambda_{n}$ is the $n$th largest eigenvalue of the reduced density matrix $\rho_{A}$. Since $0<\lambda_{n}<1$ in general, $\xi_{1} \leq$ $\xi_{2} \leq \xi_{3} \leq \cdots$.

Equation (A3) implies that $\xi_{n}$ can be considered as the eigenvalues of the entanglement Hamiltonian $H_{\mathrm{E}}$ defined as

$$
H_{\mathrm{E}}=-\ln \rho_{A}
$$

This in turn suggests that $H_{\mathrm{E}}$ can be regarded as an effective Hamiltonian to represent the density matrix $\rho_{A}$ with the Boltzmann distribution $\mathrm{e}^{-H_{\mathrm{E}}}$. Since the density matrix can be block diagonalized with respect to the number of electrons $N_{e}$ and the $z$-component $S^{z}$ of the total spin in block $A$, the entanglement spectrum $\xi_{n}$ is also labelled with these quantum numbers, i.e., $\xi_{n}=\xi\left(k, N_{e}, S^{z}\right)$, where $k(=0,1,2, \ldots)$ is an index to distinguish the entanglement spectrum in the same quantum number sector: $\xi\left(0, N_{e}, S^{z}\right) \leq \xi\left(1, N_{e}, S^{z}\right) \leq \xi\left(2, N_{e}, S^{z}\right) \leq \cdots$. We can now define the entanglement gaps for the charge sector as

$$
\begin{array}{r}
\Delta \xi_{\mathrm{C}}=\min [\xi(0, N / 2+1,1 / 2)-\xi(0, N / 2,0), \\
\xi(0, N / 2-1,1 / 2)-\xi(0, N / 2,0)]
\end{array}
$$

and for the spin sector as

$$
\begin{array}{r}
\Delta \xi_{\mathrm{S}}=\min [\xi(0, N / 2,1)-\xi(0, N / 2,0), \\
\xi(0, N / 2,-1)-\xi(0, N / 2,0)],
\end{array}
$$

where the size of block $A$ is half of the cluster size $N$.

Figure 12 shows the entanglement gaps $\Delta \xi_{\mathrm{C}}$ and $\Delta \xi_{\mathrm{S}}$ as a function of $U / t$. We indeed find that $\Delta \xi_{\mathrm{C}}$ increases abruptly at the phase boundaries. Since the value of $\Delta \xi_{\mathrm{C}}$ is related inversely to the global charge fluctuations between blocks $\mathrm{A}$ and $\mathrm{B}$, the abrupt increase of $\Delta \xi_{\mathrm{C}}$ in the phase boundary between phases I and II suggests that this transition involves the opining of charge gap. Therefore, one would regard the transition between phases I and II as the Mott transition.

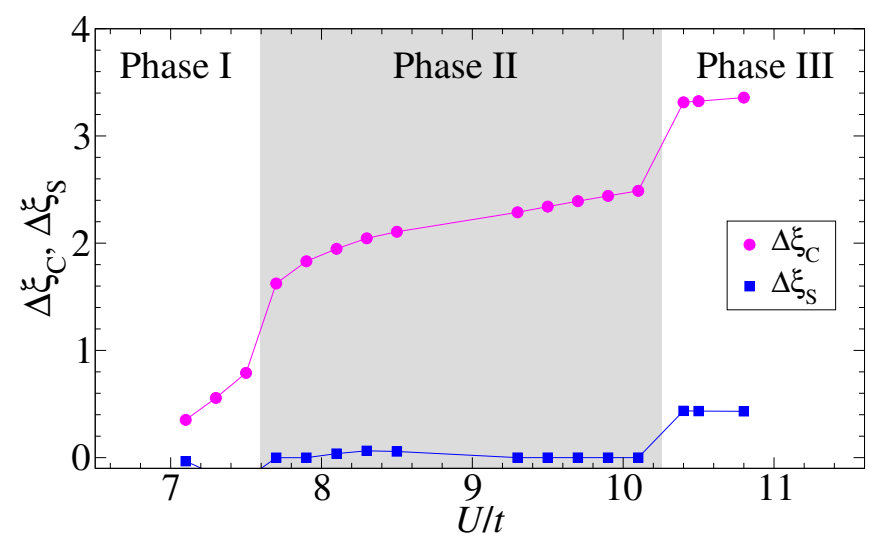

Figure 12. (color online) Entanglement gaps $\Delta \xi_{\mathrm{C}}$ and $\Delta \xi_{\mathrm{S}}$ for the charge and spin sectors, respectively. A shaded region indicates phase II determined from the discontinuities of the double occupancy in the 48 -site cluster.

We also find that the entanglement gap $\Delta \xi_{\mathrm{S}}$ for the spin sector increases abruptly at the phase boundary between phases II and III. Moreover, we find that the $\Delta \xi_{\mathrm{S}} \sim 0$ in the phase II. This tempts us to conclude that the ground state is spin gapless in phase II. However, this is not appropriate because a topologically nontrivial gapped state can induce characteristic low-lying edge states with the gapless entanglement spectrum for 
the spin sector. Therefore, we can only argue from Fig. 12 that the spin structure of phase II is distinguishable from phase III.
[1] Y. Shimizu, K. Miyagawa, K. Kanoda, M. Maesato, and G. Saito, Spin liquid state in an organic Mott insulator with a triangular Lattice, Phys. Rev. Lett. 91, 107001 (2003).

[2] T. Itou, A. Oyamada, S. Maegawa, M. Tamura, and R. Kato, Spin-liquid state in an organic spin-1/2 system on a triangular lattice, J. Phys.: Condens. Matter 19, 145247 (2007).

[3] M. Yamashita, N. Nakata, Y. Senshu, M. Nagata, H. M. Yamamoto, R. Kato, T. Shibauchi, Y. Matsuda, Highly mobile gapless excitations in a two-dimensional candidate quantum spin liquid, Science 328, 1246 (2010).

[4] S. Yamashita, T. Yamamoto, Y. Nakazawa, M. Tamura, and R. Kato, Gapless spin liquid of an organic triangular compound evidenced by thermodynamic measurements, Nat. Commun. 2, 275 (2011).

[5] T. Isono, H. Kamo, A. Ueda, K. Takahashi, M. Kimata, H. Tajima, S. Tsuchiya, T. Terashima, S. Uji, and H. Mori, Gapless quantum spin liquid in an organic spin$1 / 2$ triangular-lattice $\kappa-H_{3}(\text { Cat-EDT-TTF })_{2}$, Phys. Rev. Lett. 112, 177201 (2014).

[6] P. A. Lee, An end to the drought of quantum spin liquids, Science 321, 1306 (2008).

[7] L. Balents, Spin liquids in frustrated magnets, Nature 464, 199 (2010).

[8] P. W. Anderson, Resonating valence bonds: A new kind of insulator?, Mater. Res. Bull. 8, 153 (1973).

[9] P. Fazekas and P. W. Anderson, On the ground state properties of the anisotropic triangular antiferromagnet, Phil. Mag. 30, 423 (1974).

[10] B. Bernu, P. Lecheminant, C. Lhuillier, and L. Pierre, Exact spectra, spin susceptibilities, and order parameter of the quantum Heisenberg antiferromagnet on the triangular lattice, Phys. Rev. B 50, 10048 (1994).

[11] L. Capriotti, A. E. Trumper, and S. Sorella, Long-range Néel order in the triangular Heisenberg model, Phys. Rev. Lett. 82, 3899 (1999).

[12] W. Zheng, J. O. Fjærestad, R. R. P. Singh, R. H. McKenzie, and R. Coldea, Excitation spectra of the spin-1/2 triangular-lattice Heisenberg antiferromagnet, Phys. Rev. B 74, 224420 (2006).

[13] S. R. White and A. L. Chernyshev, Neél order in square and triangular lattice Heisenberg models, Phys. Rev. Lett 99, 127004 (2007).

[14] S. Yunoki and S. Sorella, Two spin liquid phases in the spatially anisotropic triangular Heisenberg model, Phys. Rev. B 74, 014408 (2006).

[15] O. I. Motrunich, Variational study of triangular lattice spin-1/2 model with ring exchanges and spin liquid state in $\kappa-(E T)_{2} C u_{2}(C N)_{3}$, Phys. Rev. B 72, 045105 (2005).

[16] In the context of the organic materials, other factors, e.g., the intra-dimer degree of freedom [17-19], the electronlattice coupling, and the spin-orbit coupling [7], have also been discussed.

[17] C. Hotta, Quantum electric dipoles in spin-liquid dimer Mott insulator $\kappa-E T_{2} C u_{2}(C N)_{3}$, Phys. Rev. B 82, 241104 (2010).
[18] M. Naka and S. Ishihara, Electronic ferroelectricity in a dimer Mott insulator, J. Phys. Soc. Jpn. 79, 063707 (2010).

[19] H. Watanabe, H. Seo, and S. Yunoki, Phase competition and superconductivity in $\kappa-(B E D T-T T F)_{2} X$ : Importance of intermolecular Coulomb interactions, J. Phys. Soc. Jpn. 86, 033703 (2017)

[20] Y. Kurosaki, Y. Shimizu, K. Miyagawa, K. Kanoda, and G. Saito, Mott transition from a spin liquid to a Fermi liquid in the spin-frustrated organic conductor $\kappa$ -

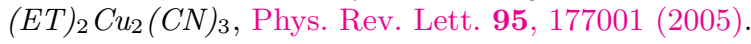

[21] H. C. Kandpal, I. Opahle, Y.-Z. Zhang, H. O. Jeschke, and R. Valentí, Revision of model parameters for $\kappa$-type charge transfer salts: An ab initio study, Phys. Rev. Lett. 103, 067004 (2009).

[22] T. Furukawa, K. Miyagawa, H. Taniguchi, R. Kato, and K. Kanoda, Quantum criticality of Mott transition in organic materials, Nature Phys. 11, 221 (2015).

[23] B. J. Powell and R. H. McKenzie, Quantum frustration in organic Mott insulators: from spin liquids to unconventional superconductors, Rep. Prog. Phys. 74, 056501 (2011).

[24] T. Koretsune, Y. Motome, and A. Furusaki, Exact diagonalization study of Mott transition in the Hubbard model on an anisotropic triangular lattice, J. Phys. Soc. Jpn. 76, 074719 (2007).

[25] R. T. Clay, H. Li, and S. Mazumdar, Absence of superconductivity in the half-filled band Hubbard model on the anisotropic triangular lattice, Phys. Rev. Lett. 101, 166403 (2008).

[26] J. Kokalj and R. H. McKenzie, Thermodynamics of a bad metal-Mott insulator transition in the presence of frustration, Phys. Rev. Lett. 110, 206402 (2013).

[27] P. Sahebsara and D. Sénéchal, Hubbard model on the triangular lattice: Spiral order and spin liquid, Phys. Rev. Lett. 100, 136402 (2008).

[28] A. Yamada, Magnetic properties and Mott transition in the Hubbard model on the anisotropic triangular lattice, Phys. Rev. B 89, 195108 (2014).

[29] H. Morita, S. Watanabe, and M. Imada, Nonmagnetic insulating states near the Mott transitions on lattice with geometrical frustration and implications for $\kappa$ $(E T)_{2} C u_{2}(C N)_{3}$, J. Phys. Soc. Jpn. 71, 2109 (2002).

[30] T. Yoshioka, A. Koga, and N. Kawakami, Quantum phase transition in the Hubbard model on a triangular lattice, Phys. Rev. Lett. 103, 036401 (2009).

[31] G. Li, An E. Antipov, A. N. Rubtsov, S. Kirchner, and W. Hanke, Competing phases of the Hubbard model on a triangular lattice: Insights from the entropy, Phys. Rev. B 89, 161118(R) (2014).

[32] H. Y. Yang, A. M. Läuchli, F. Mila, and K. P. Schmidt, Effective spin model for the spin-liquid phase of the Hubbard model on the triangular lattice, Phys. Rev. Lett. 105, 267204 (2010).

[33] T. Watanabe, H. Yokoyama, Y. Tanaka, and J. Inoue, Superconductivity and a Mott transition in a Hubbard model on an anisotropic triangular lattice, J. Phys. Soc. Jpn. 
75, 074707 (2006).

[34] T. Watanabe, H. Yokoyama, Y. Tanaka, and J. Inoue, Predominant magnetic states in the Hubbard model on anisotropic triangular lattices, Phys. Rev. B 77, 214505 (2008).

[35] L. F. Tocchio, H. Feldner, F. Becca, R. Valentí, and C. Gros, Spin-liquid versus spiral-order phases in the anisotropic triangular lattice, Phys. Rev. B 87, 035143 (2013).

[36] L. F. Tocchio, C. Gros, R. Valentí, and F. Becca, Onedimensional spin liquid, collinear, and spiral phases from uncoupled chains to the triangular lattice, Phys. Rev. B 89, 235107 (2014).

[37] B. Kyung and A.-M. S. Tremblay, Mott transition, antiferromagnetism, and d-wave superconductivity in twodimensional organic conductors, Phys. Rev. Lett. 97, 046402 (2006).

[38] T. Ohashi, T. Momoi, H. Tsunetsugu, and N. Kawakami, Finite temperature Mott transition in Hubbard model on anisotropic triangular lattice, Phys. Rev. Lett. 100, 076402 (2008).

[39] H. Lee, G. Li, and H. Monien, Hubbard model on the triangular lattice using dynamical cluster approximation and dual fermion methods, Phys. Rev. B 78, 205117 (2008).

[40] A. Liebsch, H. Ishida, and J. Merino, Mott transition in two-dimensional frustrated compounds, Phys. Rev. B 79, 195108 (2009).

[41] D. Galanakis, T. D. Stanescu, and P. Phillips, Mott transition on a triangular lattice, Phys. Rev. B 79, 115116 (2009).

[42] T. Sato, K. Hattori, and H. Tsunetsugu, Transport criticality at the Mott transition in a triangular-lattice Hubbard model, Phys. Rev. B 86, 235137 (2012).

[43] H. T. Dang, X. Y. Xu, K.-S. Chen, Z. Y. Meng, and S. Wessel, Mott transition in the triangular lattice Hubbard model: A dynamical cluster approximation study, Phys. Rev. B 91, 155101 (2015).

[44] S. Yan, D. A. Huse, and S. R. White, Spin-liquid ground state of the $S=1 / 2$ kagome Heisenberg antiferromagnet, Science 332, 1173 (2011).

[45] H.-C. Jiang, Z. Wang, and L. Balents, Identifying topological order by entanglement entropy, Nat. Phys. 8, 902 (2012).

[46] S. Depenbrock, I. P. McCulloch, and U. Schollwöck, Nature of the spin-liquid ground state of the $S=1 / 2$ Heisenberg model on the kagome lattice, Phys. Rev. Lett. 109, 067201 (2012).

[47] E. M. Stoudenmire and S. R. White, Studying twodimensional systems with the density matrix renormalization group, Annual. Rev. Condens. Matter Phys. 3, 111 (2012).

[48] H.-C. Jiang, H. Yao, and L. Balents, Spin liquid ground state of the spin-1/2 square $J_{1}-J_{2}$ Heisenberg model, Phys. Rev. B 86, 024424 (2012).

[49] S. Nishimoto, N. Shibata, and C. Hotta, Controlling frustrated liquids and solids with an applied field in a kagome Heisenberg antiferromagnet, Nat. Commun. 4, 2287 (2013).

[50] R. Ganesh, J. van den Brink, and S. Nishimoto, Deconfined criticality in the frustrated Heisenberg honeycomb antiferromagnet, Phys. Rev. Lett. 110, 127203 (2013).

[51] R. Ganesh, S. Nishimoto, and J. van den Brink, Plaquette resonating valence bond state in a frustrated honeycomb antiferromagnet, Phys. Rev. B 87, 054413 (2013).

[52] S.-S. Gong, W. Zhu, D. N. Sheng, O. I. Motrunich, and M. P. A. Fisher, Plaquette ordered phase and quantum phase diagram in the spin-1/2 $J_{1}-J_{2}$ square Heisenberg model, Phys. Rev. Lett. 113, 027201 (2014).

[53] S.-S. Gong, W. Zhu, and D. N. Sheng, Emergent chiral spin liquid: fractional quantum Hall effect in a kagome Heisenberg model, Sci. Rep. 4, 6317 (2014).

[54] S. Shinjo, S. Sota, and T. Tohyama, Density-matrix renormalization group study of the extended KitaevHeisenberg model, Phys. Rev. B 91, 054401 (2015).

[55] S.-S. Gong, W. Zhu, L. Balents, and D. N. Sheng, Global phase diagram of competing ordered and quantum spinliquid phases on the kagome lattice, Phys. Rev. B 91, 075112 (2015).

[56] D. Sellmann, X.-F. Zhang, and S. Eggert, Phase diagram of the antiferromagnetic $X X Z$ model on the triangular lattice, Phys. Rev. B 91, 081104(R) (2015).

[57] T. Tohyama, K. Tsutsui, M. Mori, S. Sota, and S. Yunoki, Enhanced charge excitations in electron-doped cuprates by resonant inelastic $x$-ray scattering, Phys. Rev. B 92, 014515 (2015).

[58] Z. Zhu and S. R. White, Spin liquid phase of the $S=1 / 2$ $J_{1}-J_{2}$ Heisenberg model on the triangular lattice, Phys. Rev. B 92, 041105(R) (2015).

[59] W.-J. Hu, S.-S. Gong, W. Zhu, and D. N. Sheng, Competing spin liquid states in the spin-1/2 Heisenberg model on triangular lattice, Phys. Rev. B 92, 140403(R) (2015).

[60] K. Shinjo, S. Sota, S. Yunoki, K. Totsuka, and T. Tohyama, Density-matrix renormalization group study of Kitaev-Heisenberg model on the triangular lattice, J. Phys. Soc. Jpn. 85, 114710 (2016).

[61] T. Okubo, K. Shinjo, Y. Yamaji, N. Kawashima, S. Sota, T. Tohyama, and M. Imada, Ground-state properties of $\mathrm{Na}_{2} \mathrm{IrO}_{3}$ determined from an ab initio Hamiltonian and its extensions containing Kitaev and extended Heisenberg interactions, Phys. Rev. B 96, 054434 (2017).

[62] S. R. White, Density matrix formulation for quantum renormalization groups, Phys. Rev. Lett. 69, 2863 (1992).

[63] S. R. White, Density-matrix algorithms for quantum renormalization groups, Phys. Rev. B 48, 10345 (1993).

[64] U. Schollwöck, The density-matrix renormalization group, Rev. Mod. Phys. 77, 259 (2005).

[65] K. Hallberg, New trends in density matrix renormalization, Adv. Phys. 55, 477 (2010).

[66] B. Normand, Frontiers in frustrated magnetism, Contemp. Phys. 50, 533 (2009).

[67] M. S. Block, D. N. Sheng, O. I. Motrunich, and M. P. A. Fisher, Spin Bose-metal and valence bond solid phases in a spin-1/2 model with ring exchanges on a four-leg triangular ladder, Phys. Rev. Lett. 106, 157202 (2011).

[68] E. H. Lieb, T. D. Schultz, and D. C. Mattis, Two soluble models of an antiferromagnetic chain, Ann. Phys. (N. Y.) 16, 407 (1961).

[69] T. Senthil, Theory of a continous Mott transition in two dimensions, Phys. Rev. B 78, 045109 (2008).

[70] R. V. Mishmash, I. González, R. G. Melko, O. I. Motrunich, and M. P. A. Fisher, Continous Mott transition between a metal and a quantum spin liquid, Phys. Rev. B 91, 235140 (2015).

[71] P. Sindzingre, P. Lecheminant, and C. Lhuillier, Investigation of different classes of variational functions for the triangular and kagomé spin-1/2 Heisenberg antiferromagnets Phys. Rev. B 50, 3108 (1994). 
[72] S. Yunoki and S. Sorella, Resonating valence bond wave function for the two-dimensional fractional spin liquid, Phys. Rev. Lett. 92, 157003 (2004).

[73] H. Li and F. D. M. Haldane, Entanglement spectrum as a generalization of entanglement entropy: Identification of topological order in non-Abelian fractional quantum Hall effect states, Phys. Rev. Lett. 101, 010504 (2008). 